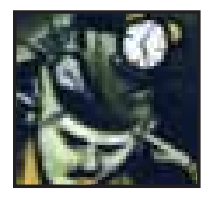

Futurethink

in HEALTHCARE

\title{
Patient/Provider Internet Connectivity: Leading the Revolution of Healthcare Delivery
}

Jeremy Nobel and Veronique Boissonnas

\section{Current Market Trends}

This article discusses the complexities of healthcare delivery and explains how the Internet is an effective platform for improving patient/provider communication and addressing many other healthcare delivery issues. The case study presented highlights how one care provider has utilized an Internet-based disease management platform to monitor and care for patients with congestive heart failure (CHF) at home.

Healthcare in the United States is transforming in fundamental ways. Recent trends in the healthcare industry include:

- Rapid consolidation of care provision

- Increased managed care penetration

- Increased focus on quality improvement and performance measurement

- A growing sense of "consumerism" among the beneficiaries of the care system

Provision of healthcare is in the process of becoming a more efficient operation as healthcare providers and payers explore new ways to become more efficient and sophisticated, and reduce costs while focusing on quality improvement and outcomes. Faced with the pressures of managed care, organizations have developed performance measurement and quality improvement tools such as disease management and care management programs that are now common in managed care and hospital settings. At the same time, patients, increasingly frustrated with the healthcare system, have started to approach their healthcare decisions with the same sense of "consumerism" with which they approach other purchases. This new era of patient involvement has increased the level of responsibility consumers take in their own care and has raised the expectations for quality improvements in healthcare delivery.

Fortunately, the trends currently transforming the healthcare delivery system have been paralleled and enabled by recent developments in Information Technology (IT). Historically the healthcare industry has been slow to adopt and invest in this type of technology. However, the Internet's low cost, ease of use and flexibility can provide the platform to support dramatic changes in the healthcare industry. Powerful IT systems are the tools needed to empower healthcare providers to do their jobs more effectively and efficiently. The development of such systems has allowed healthcare providers, managed-care organizations, employers and other players in the healthcare industry to consolidate and organize information, to use this information in clinical settings and to have speedy access to this information. IT systems have evolved from basic administrative and financial management tools to systems which have become an integral component of the care-delivery model. Use of these information systems has empowered the healthcare industry to quickly and accurately convey clinical information amongst its constituents with a "need to know"- the patient, the provider and the payer. 


\section{The Internet: A Tool in Care Management Programs}

Faced with cost-savings pressures, providers and payers have explored new interventions that will keep patients away from costly healthcare services while maintaining the quality of care. Among these new interventions is a trend towards care management programs.

The goals of care management programs are to contain costs while increasing the quality of care, providing early proactive intervention with educational programs, and preventing costly exacerbation of disease. For instance, active patient monitoring and self-management of chronic conditions has been shown to dramatically affect chronic disease outcomes (Diabetes Control and Complications Trial Research Group 1993; NIH 1991, 1993). Many studies have provided a basis for the establishment and maturation of care management programs at the patient level and disease management programs at the population level.

However to implement such programs successfully, certain challenges need to be addressed. These include enhanced communication between patients, physicians and providers and timely acquisition of data to monitor a patient's health status. This is no easy task and requires effective information management. Several technologies have emerged based on new approaches to patient/provider communication such as:

- Web-database systems

- Interactive voice response (IVR) systems

- Nurse call centers utilizing computerized systems

- Modem enabled technologies for transfer of electronic medical data from patients to healthcare providers.

Today, the Internet's ability to provide low-cost access and information exchange amongst all of the healthcare constituents (providers, payers, pharmacies and consumers) and support enhanced communication is significantly increasing the impact of such care management programs.

Healthcare electronic data interchange (EDI) started to gain momentum in the mid- to late-1980's with the automation of pharmacy claims, and later, physician claims. IT can provide even greater benefit, however, when it supports flexible information exchange, not just simple, standardizable transaction processing. In disease management, for instance, there has been an emergence of the following types of IT, each of which supports important information exchange:

- Telephone technology

- Computer networks

- "Smart" devices

Today, the Internet creates the ideal platform for disease management applications by facilitating communication between patients, providers and payers. Some of the Internet's characteristics are:
- Easy access to information

- Two-way communication

- Flexibility and timeliness

- Cross platform connectivity

- Low cost

\section{The Internet as a Care Management Communication PlatForm}

One important use of the Internet will be to create an ideal communication platform for disease management applications that improve outcomes and care management. This will be achieved by interactivity amongst patients, providers and payers, enabling and enhancing

- Rapid and accurate candidate identification

- Personalized outreach capabilities

- Interoperability with delivery environments

- Dynamic response capability

- Systematic data collection and analysis

Of note, some emerging healthcare companies already are developing and implementing Internet-enabled solutions for managed care organizations, providers and patients. These companies share a common goal: The use of improved information management strategies to solve real problems in the healthcare delivery environment. Such companies include:

- Active Health: Performs real-time multi-stream clinical data set analysis and identifies intervention opportunities. This helps providers detect important clinical situations where there may be a more effective or safer therapeutic approach available.

- NaviMedix: Provides Internet-enabled administrative and clinical workflow support at the physician's office. This allows the ambulatory office to be the hub of a communications matrix that includes the patient, the provider, the payer, pharmacies and other suppliers.

- Health Hero: Provides an Internet-based solution designed to improve the quality of care while reducing its cost. The company offers a flexible, easy-to-deploy value-added communication platform that can serve as the foundation for new disease management program or supplement and extend such programs. This allows for monitoring, education and effective intervention, leading to better medical management and outcomes assessment.

The above approaches are intriguing, inasmuch as potential benefits extend to patients, providers, and insurers. At the most basic level, these strategies significantly reduce the administrative costs associated with both care and resource management. But there are clear clinical benefits as well: 
- Patients who interact more frequently with their providers report satisfaction in managing their everyday regimen, increased convenience and self-empowerment.

- For physicians, increased data collection and communication with their patients enables them to spend more time teaching and motivating patients - thereby preventing acute disease episodes - and less time managing them.

- For insurers, financial benefits to health plans are obvious if diseases are better managed via the use of technology. Additional benefits include operational efficiency and decreased cost for collection of information.

- Other benefits include the ability to measure and survey member satisfaction and to screen for high-risk patients and help implement, enhance and focus the delivery of disease management interventions.

Care management programs typically involve the use of a wide array of information management and decision support tools to identify and assess a patient's health status and to deploy, measure and track specific strategic interventions. They also rely on technologies that leverage extensive hardware and software infrastructures, i.e., web-database systems and modem-enabled technologies for transfer of electronic medical data from patients to healthcare providers. But most importantly, these programs rely on effective and frequent communication between patients and providers, for which Internet-based infrastructure is an ideal platform.

\section{Patient/Provider Connectivity: Extending Care Management to the Patient's Home}

Chronic medical conditions are costly for society and for the individual. Of the 100 million Americans with chronic medical conditions, 12 million are unable to fully conduct normal daily activities. The cost to society reached an annual expenditure of about $\$ 1.2$ trillion in 1998. The active involvement of the patient in the treatment of these conditions promotes effective outcomes and prevents exacerbation of the chronic condition. Behavioral modification is a critical goal of the patient's self-involvement in its own care. These changes might vary from smoking cessation to participation in home monitoring and self-management of cardiovascular disease. Key elements for successfully assisting patients with selfmanagement and compliance include:

- Educating the patient about why and how to change behaviors or participate in their treatment

- Monitoring the actual degree of compliance with recommended behavioral changes

- Monitoring treatment and disease progression
- Providing motivational feedback to the patient, with rewards for compliance and notification for noncompliance

Intensive communication between the patient and the healthcare provider is one of the most important processes for affecting self-management and compliance. However, as we've discussed, communication is also the most challenging factor in a healthcare system - it is labor-intensive and expensive. It requires synchronization between the caregiver and patient. The following model describes the use of a new Internet-based disease management communication platform.

\section{ChF Disease Management Program Using Internet- Based Communication Platform: A Case Study}

A CHF disease management program was conducted by the Santa Clara County Individual Practice Association (SCCIPA), the eighth largest IPA in California including over 800 physicians responsible for 125,000 HMO members. The pilot study focused on 52 patients with $\mathrm{CHF}$ handled by one nurse care manager.

The pilot study examined the effectiveness of a disease management program utilizing an Internet-based communication platform connecting the healthcare provider directly to the patient at home. The platform leverages the Internet and advancements in microelectronics to create an improved communication infrastructure allowing a cost-effective, highly efficient, two-way communication link between caregivers and patients. This platform increases patient/provider connectivity and empowers the nurse/care manager to collect, analyze, and report on the day-to-day condition of patients in the home. It allows for quick identification of changes in patients' health conditions and fosters management by exception, thereby focusing on those patients in need of immediate intervention.

\section{Program Objectives}

On the caregiver side, the objective was to determine the effectiveness of a disease management program using an Internet-based communication platform and how the technology enables the care provider to:

- Track weight, blood pressure, edema and shortness of breath

- Track diet and medication compliance

- Facilitate timely follow-up care:

- Appointments/screenings

- Medications and lab results

- Advise and educate each patient every day

- Use on-call advice triage nights and weekends

- Encourage wellness classes

From the patient side, this program was developed as very personal and engaging to promote their involvement in their own care and by evaluating such parameters as: 
- Self-monitoring of vital signs

- Medication and program compliance

- Better involvement and participation in one's own care

- Education and information

\section{How the Health Hero Internet-Based Communication Platform Works}

Each patient receives a personal information appliance that he or she simply plugs into a phone line. The design of the appliance is the result of extensive evaluation with diverse age groups including young, elderly and disabled patients. The device is extremely easy-to-use and is designed for achieving high patient compliance, especially to a senior population.

The appliance gathers quantitative and qualitative patient information through simple question-and-answer sessions that are transmitted to the device over normal telephone lines from a data center. The patients view questions and information on a brightly lit, high-contrast liquid crystal display, and respond via four large buttons. Once the device is plugged into both electrical and phone outlets, it begins by walking the patient through a brief, repeatable training exercise designed to give the patient an opportunity to practice answering the range of questions they will encounter in their sessions. When it is properly connected, the device will prompt the patient that everything is okay and instruct the patient to check back for the first session per instructions given by the care manager. That evening, the appliance will re-connect to the network, the day's session will be downloaded, and the device will be ready for patient interaction.

To load and transmit patient information, the appliance calls the data center via a toll-free telephone number. The data center, which houses the website and database servers, is developed in conjunction with and managed by EDS. The data center is a distributed data management system designed to handle 2,000,000 patient connections daily. The central patient database is online, storing all patient updates and metric data, and is accessible only by authorized medical personnel. The data center uses both the Netscape Enterprise web server and the BEA Weblogic application server to serve HTTPS pages. All data is encrypted to the 128-bit RSA standards. In accordance with industry standards, the hardware, software and database are designed with appropriate redundancy and high security. The service provider and the data center's operations staff handle high-level account administration. Clients are responsible for their own system administration (primarily creating and updating patient and client account information).

The data center is programmed to transmit patient information collected during the previous day and receive new information and questions for the coming day. The question types include multiple choice, extended multiple choice, range, numeric, prompt, escalate, read meter and reminders. This information and all patient medical data are transmitted between the service data center and the user's PC using two different protocols. User ID's, passwords, and patient reports are transmitted as Web pages using HTTPS protocol. Webbased applications used by the care manager to interact with the patients communicate with the data center using Enterprise Java Beans (EJB) via the Secure Socket Layer (SSL) protocol. The care manager accesses these applications through a password-protected website using Explorer or Netscape Navigator web browser software. The web-based applications allow him or her to track patient status via an Internet connection and collect information, analyze patient's health status, and report on the day-to-day condition of patients in the home. Formal training is available to instruct in the use of these web-based tools and the website. Additionally, online documentation, training, Frequently Asked Questions (FAQ) and technical tips are accessible 24 hours through the website.

Specific applications currently available include:

- Care Administrator ${ }^{\mathrm{TM}}$ : This application enables care managers to enroll a patient, initiating an automatic fulfillment of a patient's appliance and performing other routine administrative functions

- Care Director ${ }^{\mathrm{TM}}$ : This application enables an authorized care manager or physician to access patient data and send queries to patients. This provides a view of the patient population, stratified by risk, sorting patient data daily. This allows the care manager to identify those patients who are currently at risk or who require attention.

- Care Composer ${ }^{\mathrm{TM}}$ : This application enables the care manager to write or adapt queries to their own specific requirements or to specific disease states and store them in the data center.

More specifically, these applications enable the care manager to: - Easily query patient populations creating their own personalized questionnaire or template using Care Composer ${ }^{\mathrm{TM}}$

- Analyze patient responses and alerts and customize sessions for individuals using Care Director ${ }^{\mathrm{TM}}$

- Review patient trends and receive notifications of escalation or failure of patient response. All online patient results can be retrieved individually or in aggregate form across groups of patients for viewing and further analysis in tables and graphs.

- Present population and patient reports to physicians. Reports can be stored as files or printed after creation. The care manager communicates specific clinical parameters/events to primary care practitioners and cardiologists by phone, fax and/or email. Additionally, patient response data, including dialogues, questions and answers, and metric data, can be exported from the system. IT programs can be developed, by 
or for customers or licensees, to export patient results for integration into an off-line database. Patient confidentiality is protected through rigorous authorization and validation, and data encryption.

\section{How the CHF Program Works}

Traditionally, patient care is based on episodic encounters between patients and their care providers. Working from the website, the SCCIPA care manager interacts with patients by assigning daily, pre-defined queries customized to the patient's needs. The dialogue used by SCCIPA includes questions related to heart failure and queries about the patient's functional health status. The software sorts patients' responses according to risk levels defined by the caregiver. Color coded risk alerts allow the provider to, at-a-glance, quickly determine if a patient needs immediate intervention. One advantage of this system is that if CHF patients enter information correctly and there are no abnormal readings, the nurse does not need to call the patients. This leaves more time for the nurse to spend with patients who really need the one-on-one assistance.

Technology-driven disease management programs, leveraging the power of the Internet, provide key value components such as:

- Cost-effectiveness approach

- Reliable assessment tools

- Centralized data management and retrieval

- Patient follow-up

- Management by exception

\section{Study Preliminary Results}

The participants were asked about:

- Their attitudes and opinions regarding the maintenance of their health and living with $\mathrm{CHF}$

- Their self-management behavior

- Their knowledge of self-management

- Their satisfaction with the personal information appliance

The preliminary evaluation was based on data for compliance and patient satisfaction collected in August 1999 through telephone interviews, site visits and data analysis. In summary, this study suggests that increased communication between patients and providers can improve patient's perceived quality of life and compliance to a sometimes very demanding health program.

Compliance: The data collected shows that patients who plug their device in the phone line and start using the appliance use it almost everyday, missing about one day in nine on average. In addition, the study collected information on patient's compliance to specific CHF diet (Figure 1) and showed a real improvement in patients' overall compliance to their diet.
Compliance is defined in the study by the number of days that a non-hospitalized patient or a group of patients answered the survey (Figure 2). Compliance is very important to measure, as it in fact may compromise outcomes if a patient doesn't respond. The absence of response makes it difficult for a care manager to identify problems early on and prevent hospitalizations. Furthermore, low compliance may increase care management costs due to increased number of interventions necessary to manage the patient.

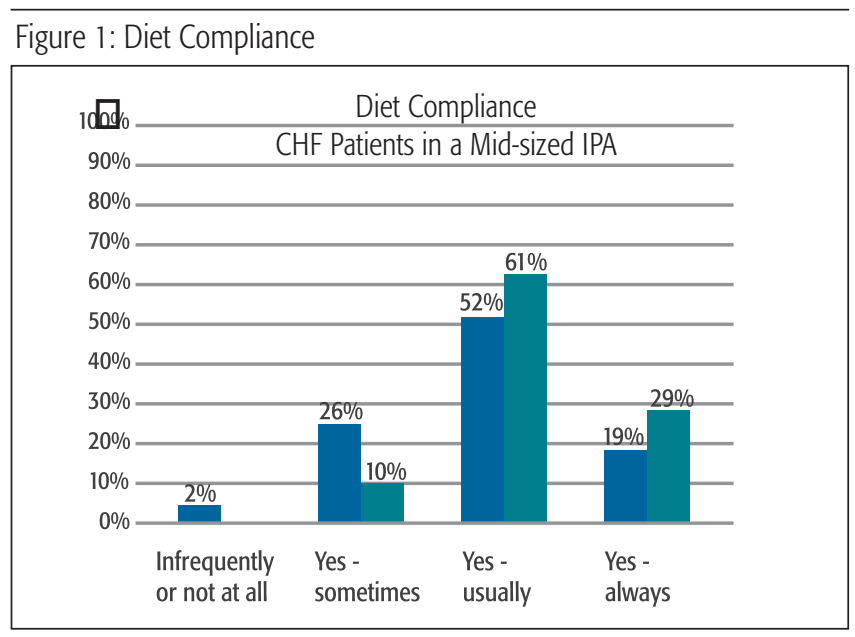

Figure 2: Compliance Evaluation

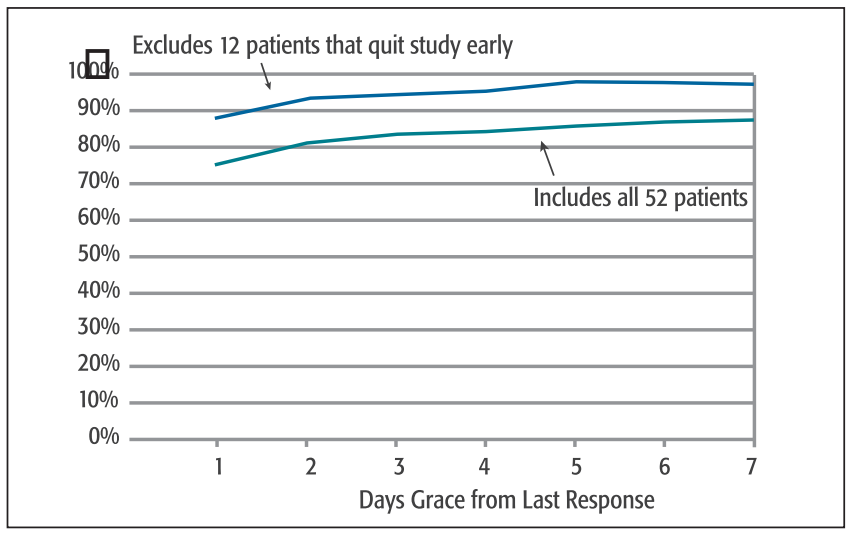

Patient Satisfaction: The result of the evaluation showed that $93 \%$ of patients have a much or somewhat better understanding of heart failure and how to manage the condition (Figure 3). Additionally, $88 \%$ of patients feel much or somewhat more confident in taking care of their condition as a results of the program (Figure 4).

Although data has not yet been collected for medical outcomes and cost-savings for this specific case study, other studies have demonstrated that remote patient monitoring facilitates earlier interventions and eliminates emergency visits and hospitalizations and decreases cost of care. For instance, a 


\section{SUBSCRIBE NOW}

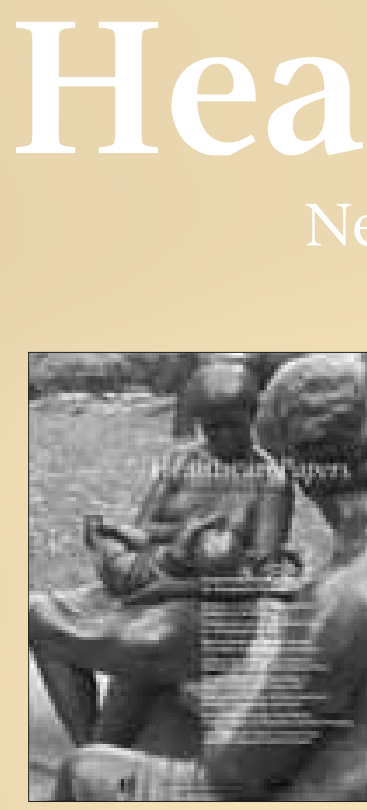

Primary Care

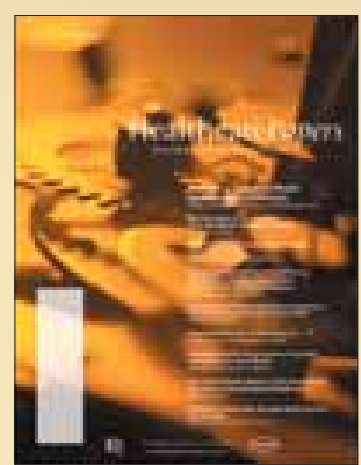

Integrated Healthcare

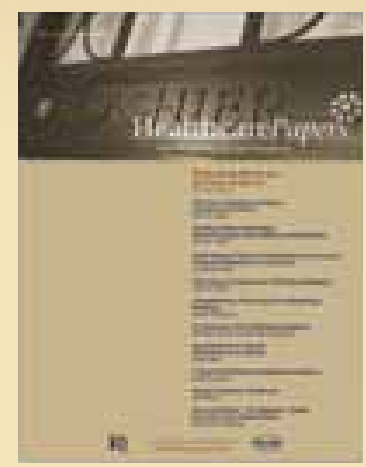

Rethinking Medicare

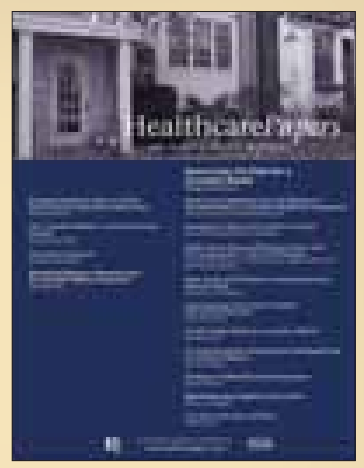

Home Care

$$
\begin{array}{ll}
\text { coming } & \text { - Mitigating Medical Errors } \\
\text { - Rationing at the Bedside } \\
\text { - Evolution of Academic Health Sciences Centres } \\
\text { - Human Resources Strategies } \\
\text { - One Tier/Two Tier } \\
\text { - Pharmacare and the Drug Trade } \\
\text { - Retooling/Recapitalizing }
\end{array}
$$

\section{FOR FASTER SERVICE}

\section{www.healthcarepapers.com}

\section{Toronto 416 864-9667}

or email Barbara Marshall at: bmarshall@longwoods.com

Subscriptions going to a Canadian address are sold in Canadian dollars. Subscriptions going outside of Canadian borders are due in US dollars.

This includes postage and handling. In Canada, please add 7\% CST where applicable. Our CST\# is R138513668

\section{SUBSCRIPTION ORDER FORM}

YES, I want to subscribe to the next 4 issues of HealthcarePapers for \$125 + CST

Name Title

Organization

Address

City

Province/State

Postal/ZIP Code

Telephone

Facsimile

Email

You can mail this form to:

Send me an invoice

Longwoods Publishing Corp., 260 Adelaide Street East, Box 8, Toronto, Ontario, M5A 1N1 Canada

Cheque enclosed

(payable to HealthcarePapers)

VISA

...or fax it to 416 368-6292

Card \#

Signature

Expiry Date 
Figure 3: Patient's Understanding of Heart Failure and how to Manage the Condition

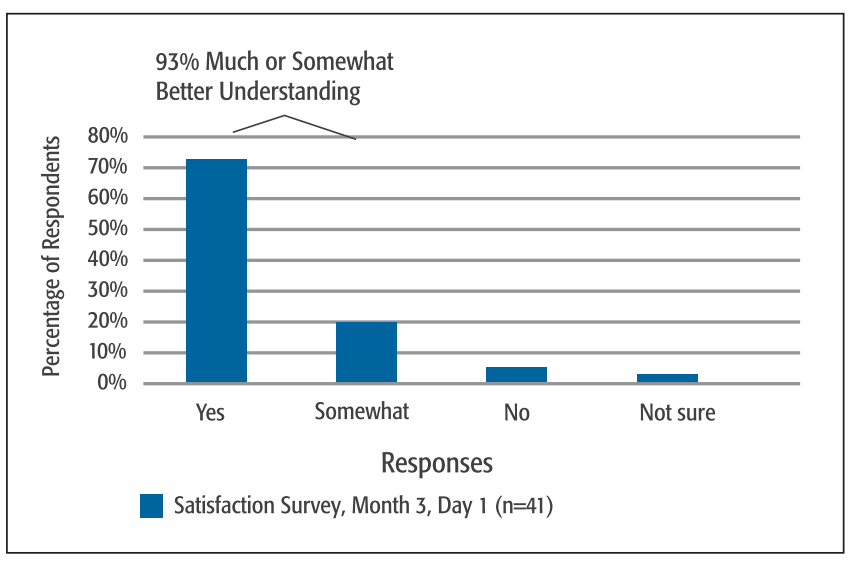

Figure 4: Patient's Feeling About Self-Care

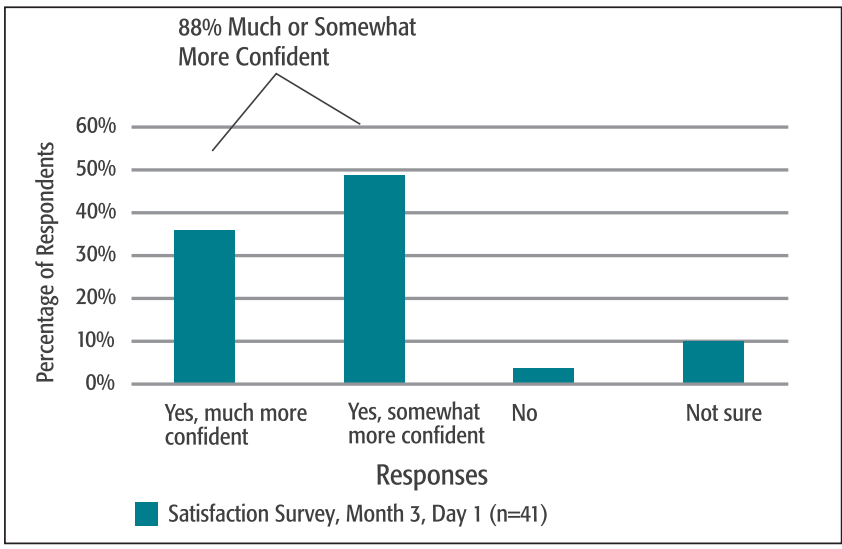

Pediatric Asthma Self-Management Program (Reissiger et al. 1998), combining education, nursing assessment and series of surveys reported:

- $61 \%$ reduction in hospitalization

- 46\% reduction in physicians visits

- Estimated annual savings of more than $\$ 11,500$ per patient

Another congestive heart failure home monitoring program using patient education, daily self-monitoring, and physician notification for abnormal weight gain, vital signs, and symptoms reported:

- $85.5 \%$ daily compliance rate per patient

- $66 \%$ reduction in ER visits

- $73 \%$ reduction in Intensive Care Unit admissions

\section{CONCLUSION}

Disease management programs leveraging an Internet-based population management platform empower healthcare providers to collect, analyze and report information on the day- to-day conditions of patients in their home. This technology also enables healthcare providers to quickly focus on patients in need of intervention and assure compliance with health regimens, which ultimately results in better outcomes at lower costs to the healthcare system. Additionally, patients have a better understanding of their condition and how to manage it.

In such scenarios, care management programs allow for the collection and analysis of medical data, such as pre-visit screenings, laboratory results, prior to the patient's visit, thereby enabling the care provider to spend more time interacting with and educating the patient. Further, the physician can have a printed report of the patient's health status and history so that the time with the patient can be spent teaching and intervening rather than data collecting.

Utilizing technology-driven disease management and care management systems facilitates a care manager's ability to provide proactive healthcare at a low cost and responds to healthcare's major challenges. Web-enabled technologies, as presented in the case study, enhance effective communication between all healthcare constituents, promoting an interactive exchange of information. Increased patient/provider connectivity enables care managers to monitor patients more efficiently, thereby increasing productivity and reducing crises for patients. These benefits can be translated to dollar savings for the healthcare industry and better outcomes for patients. IQ

\section{REFERENCES}

The Diabetes Control and Complications Trial Research Group. 1993. "The effect of Intensive Treatment of Diabetes on the Development and Progression of Long-Term Complications in Insulin-Dependent Diabetes Mellitus." N England J Med 329: 977-86.

National Institutes of Health. 1991. National Heart, Lung and Blood Institute. Executive Summary: Guidelines for the Diagnosis and Management of Asthma. Publication 91-3042A.

National Institutes of Health. 1993. National High Blood Pressure Education Program. Working group report on primary prevention of hypertension. Publication 93-2669.

Reissiger, A., D. Schlumper, W. Wyckoff. Olsten Health Services. 1998. "A Pediatric Asthma Self-Management Program That Gets Results." Drug Benefits Trends 10(1): 24-26

Jeremy Nobel, MD, MPH, is a course director in the Harvard School of Public Health. He also designs healthcare management and delivery systems. His particular interest is in the development of computer based information technology applications that coordinate critical aspects of healthcare delivery.

Veronique Boissonnas is a healthcare communications consultant at Noonan/Russo Communications. She specializes in managed care and e-health communications.

COPYRIGHT @ 2000 BY THE HEALTHCARE INFORMATION AND MANAGEMENT SYSTEMS SOCIETY. 


\section{Commentary: The Grass is Covered in Snow}

\section{BenJAMin BurKo}

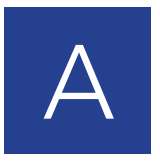
lthough Canada's socialized, one payer healthcare system is sharply different from its managed-care counterpart in the United States, physicians and patients largely experience similar problems in their day-to-day interactions with each other. In public survey after survey, Canadians state that healthcare is the number one public issue on their agenda. To that end, the Canadian government has earmarked over $\$ 500$ million for electronic and internet-based programs which increase access to healthcare and the quality of healthcare service delivery. The public is ready, but are physicians?

In a national study of approximately 450 Canadian physicians completed in August-September 2000, it was determined that the participating physicians used the Internet to:

- gather information about drugs - 73\%

- look up treatment protocols - $61 \%$

- consult with colleagues - $42 \%$

Regarding where Canadian physicians use the Internet:

- $57 \%$ report having online access both at work and at home

- $35 \%$ have access exclusively at home

- $6 \%$ say they only have access at work.

Specialists (66\%) were more likely than family doctors $(46 \%)$ to have Internet access at both home and work.

When it comes to issues of practice management and communicating with patients, Canadian and American physicians are very different from each other. In fact, only $16 \%$ of Canadian physicians claimed to want to use the Internet to write on-line prescriptions, and 55\% clearly stated they did not want to use the Internet for this purpose. Similarly, 15\% of Canadian MD's expressed an interest in sending e-mail to patients, whereas $49 \%$ firmly expressed a lack of desire to do so. In areas that have been more readily accepted for computerization by Canadian physicians - namely billing services $39 \%$ report they have little or no interest in filing billing information online compared to $31 \%$ who report they would be very or extremely interested in such a service. Approximately 19\% of respondents say they currently use the Internet to file billing information.

An area of great potential interest to patients is the ability to use the Internet to participate in clinical trials. This would involve sending targeted patients, who have a certain disease or profile, an invitation to participate in a trial that may ultimately benefit patients just like themselves. Only $34 \%$ of responding physicians said they have a great interest in this service while $24 \%$ report little to no interest.

Is money the ultimate motivator in getting Canadian physicians to use the Internet? Apparently not. When asked: "If provincial governments covered the service, would you provide consults by e-mail?" Forty percent of doctors still said they would not, while $45 \%$ said they would. In fact, a full $46 \%$ of all respondents felt that Internet use would decrease their incomes while only $5 \%$ felt that medical Internet use could increase their incomes.

Despite the fact that the United States has developed a model of managed care, with the stated goals of improving quality of care and decreasing costs, it still spends a far greater proportion of its GDP on healthcare than its Canadian neighbours to the north. (In 1998, US portion of GDP for healthcare was $14.0 \%$ while in Canada it was $9.3 \%$; OECD 2000). US physicians have greater access to computers in their workplace and to these Internet-based tools. One must question whether Canadian physicians have been slower to embrace these tools because, even in the absence of "managed care," they are already better managed, and the needs are less pressing. Or perhaps their attitude is a result of negative attitudes which prevail and prevent wider spread usage. The challenge remains to align all of the constituencies around a secure, efficient, economical internet-based healthcare strategy for the common good. IQ

\section{REFERENCES:}

Canada Health Infoway. 1999. Paths to Better Health: Final Report. Advisory Council on Health Infostructure.

The Medical Post. 2000. National Survey of Doctors. Rogers Media. October.

OECD. 2000. Health Data 99. Paris: Organization for Economic Cooperation and Development.

Dr. Benjamin Burko, CEO StealthMD Corporation, is also an assistant professor of Medicine at the McGill University Health Centre and Medical Director of Tiny Tots Medical Centre.

Telehealth never waits! Why shourd you?

Subscribe to Busy Signals: a FREE, bi-weekly,

electronic publication covering Tel/E-Health news

in Canada and around the world.

Go to http://www.cyberhealth.bc.ca/signals

or E-mail editor@cyberhealth.bc.ca 